\title{
Arithmetic operation signs elicit spatial associations: A confirmatory Bayesian analysis
}

\author{
Keelyn Brennan ${ }^{1}$, Michaela Rutledge ${ }^{1}$, \& Thomas J. Faulkenberry ${ }^{1}$ \\ 1 Tarleton State University
}

\begin{abstract}
Previous research in numerical cognition has indicated that people form mental associations between numbers and space. The purpose of the present study was to replicate and confirm an operation sign spatial association originally reported by Pinhas, Shaki, and Fischer (2014). In our study, participants were asked to make a speeded classification of the arithmetic operation (addition or subtraction) that was represented by two mathematical operation signs ( + or -$)$. Bayesian analysis confirmed that response times increased in the condition where response codes were incongruent with an "addition $=$ right side of space / subtraction = left side of space" mapping. These results confirm those originally reported by Pinhas et al. (2014) and indicate that people may form an association between arithmetic signs and spatial location. Further, we demonstrate how to perform a confirmatory Bayesian analysis in the free software package JASP, including the use of sensitivity analysis and informed priors. In addition to explaining how spatial-operational associations are a potentially fruitful line of inquiry in numerical cognition, the present work provides a concise demonstration of how to conduct a confirmatory Bayesian analysis.
\end{abstract}

Keywords: Operation signs, spatial association, Bayes factor, informed priors, JASP

Research in numerical cognition is primarily concerned with questions about how numbers are represented in the mind and brain. Much previous research in this field has indicated that people form spontaneous mental associations between numbers and space (Faulkenberry, 2016; Fischer \& Shaki, 2014; Marghetis, Núñez, \& Bergen, 2014). The

This work was supported in part by a Faculty-Student Research Grant from Tarleton State University awarded to TJF. The raw data and JASP analysis file are available for download at https://osf.io/4sedf/.

Correspondence concerning this article should be addressed to Thomas J. Faulkenberry, Department of Psychological Sciences, Box T-0820, Tarleton State University, Stephenville, TX 76401. E-mail: faulkenberry @tarleton.edu 
purpose of the present study is to investigate one such association - a spatial association with arithmetic operation signs (Pinhas et al., 2014) - and perform a confirmatory Bayesian analysis of the observed data.

These spatial-numerical associations are typically revealed in the form of increased response times (RTs) on experimental trials with stimuli that are in an opposite configuration to the proposed association. For example, consider a task where people are asked to classify the parity (i.e., even or odd) of presented number digits. In one half of the experimental trials, participants indicate even digits with a left hand button press and odd digits with a right hand button press. In the other half, these instructions are switched, thus ensuring that every number digit is responded to with both hands throughout the course of the experiment. Even though a judgment of number magnitude (i.e., small, large) is not required for the task, number magnitude seems to interact with response hand. That is, people are faster to respond to small numbers with the left hand and large numbers with the right hand. This phenomenon is called the SNARC effect (Dehaene, Bossini, \& Giraux, 1993; Dehaene, Dupoux, \& Mehler, 1990), where SNARC is an acronym for Spatial-Numerical Associations of Response Codes. Because RTs are longer when response codes are opposite the typical "left = small, right = large" orientation of the physical number line, these data are widely thought to represent a left-to-right spatial ordering of the mental number line.

Remarkably, this left-to-right spatial association with numbers has been found in mental arithmetic as well. McCrink, Dehaene, and Dehaene-Lambertz (2007) had participants perform a nonsymbolic arithmetic task. For addition problems, two dot patterns moved behind a blocking screen; one traveled from the left side of the screen and one traveled from the right side of the screen). Afterward, the screen was removed and participants were asked to judge whether the number of dots revealed was the correct outcome. Subtraction problems were performed similarly; at first a dot pattern traveled from the left to move behind the screen, then a subset of dots continued the implied trajectory and moved away to the right (effectively "taking away" from the original dot collection). Again, the screen was removed, and participants were asked to judge the correctness of the remaining dot pattern. Error patterns on this task indicated that participants overestimated their answers to addition problems, whereas subtraction problems led to underestimation. McCrink et al. (2007) interpreted these error patterns as an operational momentum effect, reflecting an "overshooting" of the correct answer as participants scanned along the mental number line (rightward for addition, leftward for subtraction).

Though one might argue that this operational momentum is an artifact of the nonsymbolic arithmetic task that McCrink et al. (2007) used, similar results appear even in symbolic tasks involving just an operation sign. For example, Pinhas et al. (2014) had participants perform a simple operation sign classification task, identifying whether a presented operation $\operatorname{sign}(+$ or -$)$ represented the operation of addition or subtraction, respectively. On half of the trials, participants responded "addition" with a right-hand button press, and "subtraction" with a left-hand button press. On the other half of trials, this response mapping was reversed. Pinhas et al. (2014) discovered that people are faster to classify the "+" sign in the "addition = right hand" condition, and similarly, faster to classify the "-" sign in the "subtraction = left hand" condition. Because RTs were increased in the condition where response codes 
were opposite (i.e., incongruent) with an "addition = right side of space / subtraction = left side of space" mapping, Pinhas et al. (2014) interpreted these data as reflecting an inherent spatial-arithmetical association and coined the association as the OSSA effect (operation sign spatial association).

The Pinhas et al. (2014) result is truly remarkable, as it indicates that spatialarithmetical associations exist at a fundamental level. Indeed, it seems that the presence of a lone addition sign can promote a rightward spatial momentum. Thus, these data seem to indicate that people possess an inherent spatial association with the operation sign itself, and the context of actually performing mental arithmetic is not even necessary to evoke the effect. To our knowledge, the OSSA effect of Pinhas et al. has not been independently replicated. Replication is necessary in order to justify any claims of spatial-arithmetical association, as such claims have become a fundamental component in the recent literature on the nature of mental arithmetic (Azhar, Chen, \& Campbell, 2020; Chen \& Campbell, 2017; Thevenot \& Barrouillet, 2020).

In the present study, we attempted to replicate the OSSA effect of Pinhas et al. (2014). Additionally, we performed a fully confirmatory Bayesian analysis of the resulting data (Faulkenberry, Ly, \& Wagenmakers, 2020). In a Bayesian analysis, we are able to mathematically specify competing hypotheses about the effect sizes we expected to observe in our experiment and then measure the relative predictive performance of each hypothesis (i.e., the evidence for each hypothesis). This evidence is quantified with a Bayes factor (Faulkenberry, 2022; Kass \& Raftery, 1995; Wagenmakers, 2007), which measures the extent to which the observed data are more likely under one hypothesis compared to the other. This approach is growing in popularity in the psychological sciences, both for its conceptual simplicity and for its pragmatic solution to the problems inherent in traditional hypothesis testing with p-values (Wagenmakers, 2007). With Bayesian analysis, there is no need to choose between hypothesis testing and estimation (i.e., Cumming \& Calin-Jageman, 2016). Since both are integrated into the same analysis, we can first assess the evidence for (or against) the OSSA effect, and if we find positive evidence for the effect, we can then estimate the size of the effect and quantify the uncertainty of this estimate. Additionally, the Bayesian approach gives us the ability to test the robustness of our claims against different prior settings as well as track the impact of collecting additional subjects on accumulated evidence.

\section{Method}

\section{Participants}

We tested 35 participants ( 28 female, mean age $=22.8$ years, age range 19 to 61 years) on an operation sign classification task. All 35 participants were students at Tarleton State University who participated in exchange for partial course credit in an introductory level psychology course.

\section{Design and procedure}

Participants began the experimental session by sitting approximately $60 \mathrm{~cm}$ in front of a computer. After signing a consent form and completing a brief demographic questionnaire, each participant then completed an operation sign classification task. The task was 
programmed and deployed using SuperLab 5.0 software on a 20-inch iMac desktop computer with a screen resolution of $1280 \times 1040$ pixels. Participant input was captured using a Cedrus Corporation RB-730 response pad.

During the task, the arithmetic operation signs "+" and "-" were presented as stimuli. Participants were asked to classify each presented operation sign as representing either addition or subtraction. These stimuli were presented in 60-point Courier font in black on a gray background $($ RGB color values red $=153$, green $=153$, blue $=153$ ). Participants were instructed to keep their left and right index fingers on the leftmost and rightmost buttons (respectively) of the response pad. Once a brief instruction page was read, the participant initiated the first of the experimental trials with a button press. Each trial began with a blank screen presented for $750 \mathrm{~ms}$, followed by a randomly selected operation sign $(+$ or -) that remained on the center of the screen until a response was made. The computer displayed a red "X" after an error, and no feedback was given for correct responses.

Participants completed the task under two counterbalanced response rules. On half of the trials, participants were asked to respond "addition" with a right-hand button press and "subtraction" with a left-hand button press (congruent response rule). On the other half of the trials, this response rule was reversed (incongruent response rule). Eighteen participants began with the congruent response rule, whereas 17 began with the incongruent response rule. Each sign was presented 14 times in random order for a total of 28 repetitions per response rule. The response rule was switched after the completion of these 28 trials and the procedure was repeated for the other response rule. In all, each participant completed a total of 56 trials of the operation sign classification task.

\section{Results}

\section{Data preparation}

Participants completed a total of 1,960 experimental trials. We removed 87 error trials (4.4\% error rate), as well as 12 trials ( $0.64 \%$ of remaining trials) for which RT was less than $200 \mathrm{~ms}$ or greater than $2000 \mathrm{~ms}$. Each trial was then classified as either congruent or incongruent according to response rule. Congruent trials were those on which either the addition sign was responded to with the right hand or the subtraction sign was responded to with the left hand. All other trials were classified as incongruent. The remaining RTs were collapsed (via the mean) into 70 design cells, obtained by crossing the factors of participant $(N=35)$ and condition (congruent, incongruent).

\section{Bayesian hypothesis testing}

We chose to analyze our data with the default Bayesian $t$-test (Faulkenberry, 2019a; Rouder, Speckman, Sun, Morey, \& Iverson, 2009). The Bayesian $t$-test works by first specifying two competing hypotheses about the population-level effect size $\delta$. This effect size reflects the standardized mean difference in RTs between congruent and incongruent trials. Since past studies indicate that RT should increase on incongruent trials, we too expected to find $\delta>0$. Thus, we formally defined our two competing hypotheses to test this expectation. The null hypothesis was defined as an effect size of 0 , denoted $\mathcal{H}_{0}: \delta=0$, and the alternative hypothesis was defined to be a positive effect, denoted $\mathcal{H}_{+}: \delta>0$. 
To perform a Bayesian analysis, we must assign two types of prior belief before observing data: (1) the prior model probabilities of the two hypotheses $\mathcal{H}_{0}$ and $\mathcal{H}_{+}$; and (2) the prior distribution of effect sizes $\delta$ that we expect under $\mathcal{H}_{+}$. We used default specifications for both. To define the prior model probabilities, we set the prior probability of each hypothesis to $p\left(\mathcal{H}_{0}\right)=p\left(\mathcal{H}_{+}\right)=0.50$, giving us 1-to- 1 prior odds and indicating no a priori preference for either hypothesis before observing data. Second, we set the prior distribution on $\delta$ as a positive half-Cauchy distribution with scale $1 / \sqrt{2}=0.707$ (see the left panel of Figure 1). This prior scale setting means that before observing any data, we expect with probability $1 / 2$ that the latent population-level effect size $\delta$ should have magnitude less than 0.707. The number 0.707 (called the scale of the Cauchy distribution) is chosen because the resulting prior on $\delta$ has a number of desirable mathematical properties (Rouder et al., 2009).
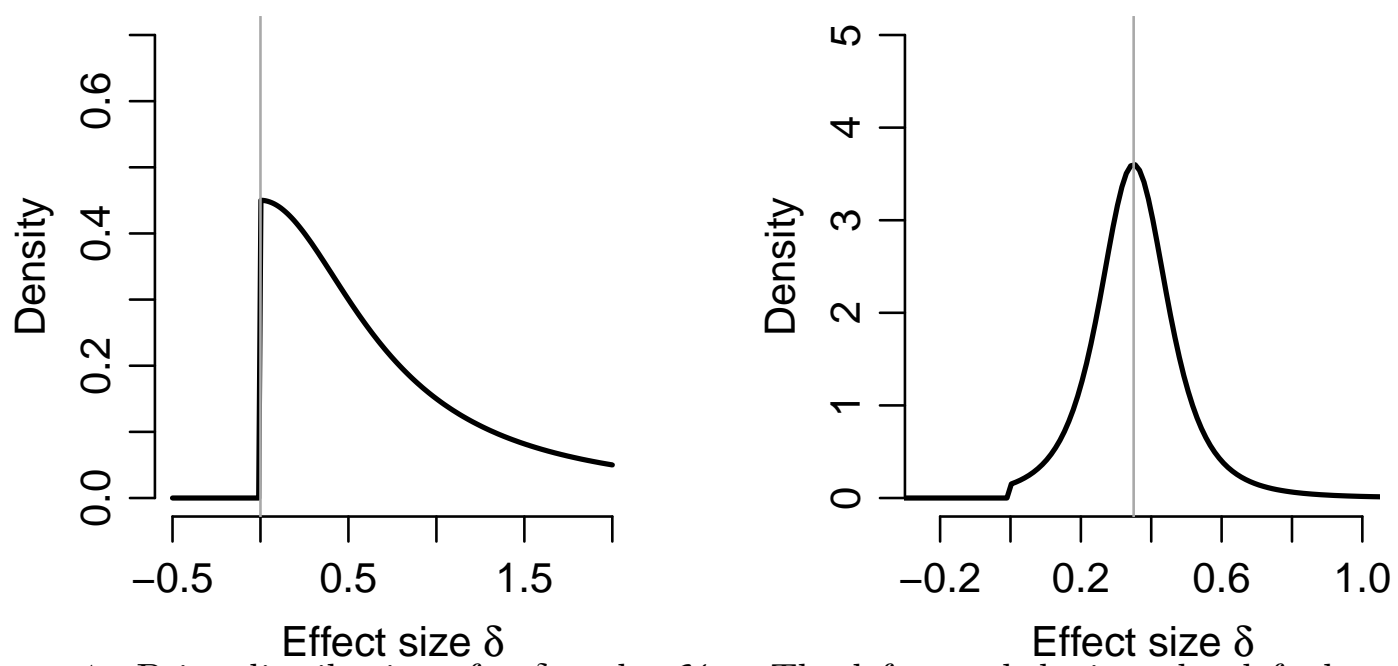

Figure 1. Prior distributions for $\delta$ under $\mathcal{H}_{+}$. The left panel depicts the default prior, defined as a positive half-Cauchy distribution with scale $1 / \sqrt{2}$. The right panel depicts the informed Oosterwijk prior, defined as a scaled noncentral $t$-distribution with center 0.35 , scale 0.102 , and $d f=3$.

Model comparison was then performed using the Bayes factor (Faulkenberry, 2022; Kass \& Raftery, 1995), which indexes the relative likelihood of observing our data under $\mathcal{H}_{0}$ and $\mathcal{H}_{+}$. For example, $\mathrm{BF}_{+0}=9$ would mean that our observed data are 9 times more likely under $\mathcal{H}_{+}$than $\mathcal{H}_{0}$; this would show positive evidence for $\mathcal{H}_{+}$. On the other hand, $\mathrm{BF}_{+0}=1 / 9$ would mean that $\mathrm{BF}_{0+}=1 / \mathrm{BF}_{+0}=1 /(1 / 9)=9$, indicating that the data is 9 times more likely under $\mathcal{H}_{0}$ than $\mathcal{H}_{+}$, thus providing positive evidence for $\mathcal{H}_{0}$. Bayes factors were then converted to posterior model probabilities for $\mathcal{H}_{0}$ and $\mathcal{H}_{+}$(Faulkenberry, 2019b; Masson, 2011) using the following equation:

$$
p\left(\mathcal{H}_{+} \mid \text {data }\right)=\frac{\mathrm{BF}_{+0}}{1+\mathrm{BF}_{+0}} .
$$

These posterior model probabilities give us another easy-to-interpret index of model preference. The hypothesis with posterior probability greater than 0.50 is the preferred 
model, and the closer the posterior probability is to 1 , the greater our preference for that model. All Bayes factors reported below were computed using the free software package JASP (Faulkenberry et al., 2020; JASP Team, 2020), which can be freely downloaded from https://www.jasp-stats.org.

\section{Response time analysis}

Mean response times for each experimental condition can be seen in Figure 2. As is immediately clear, responses on congruent trials were faster than responses on incongruent trials. When the presented stimulus was a plus sign ("+"), responses with the right hand were faster $(M=439 \mathrm{~ms})$ than responses with the left hand $(M=483 \mathrm{~ms})$. When the presented stimulus was a minus sign ("“"), the pattern was opposite - responses with the left hand were faster $(M=446 \mathrm{~ms})$ than responses with the right hand $(M=474 \mathrm{~ms})$. In both cases, the means reflect the operation sign spatial association (OSSA) of (Pinhas et al., 2014). We will now assess the evidence for this effect with a Bayesian $t$-test.

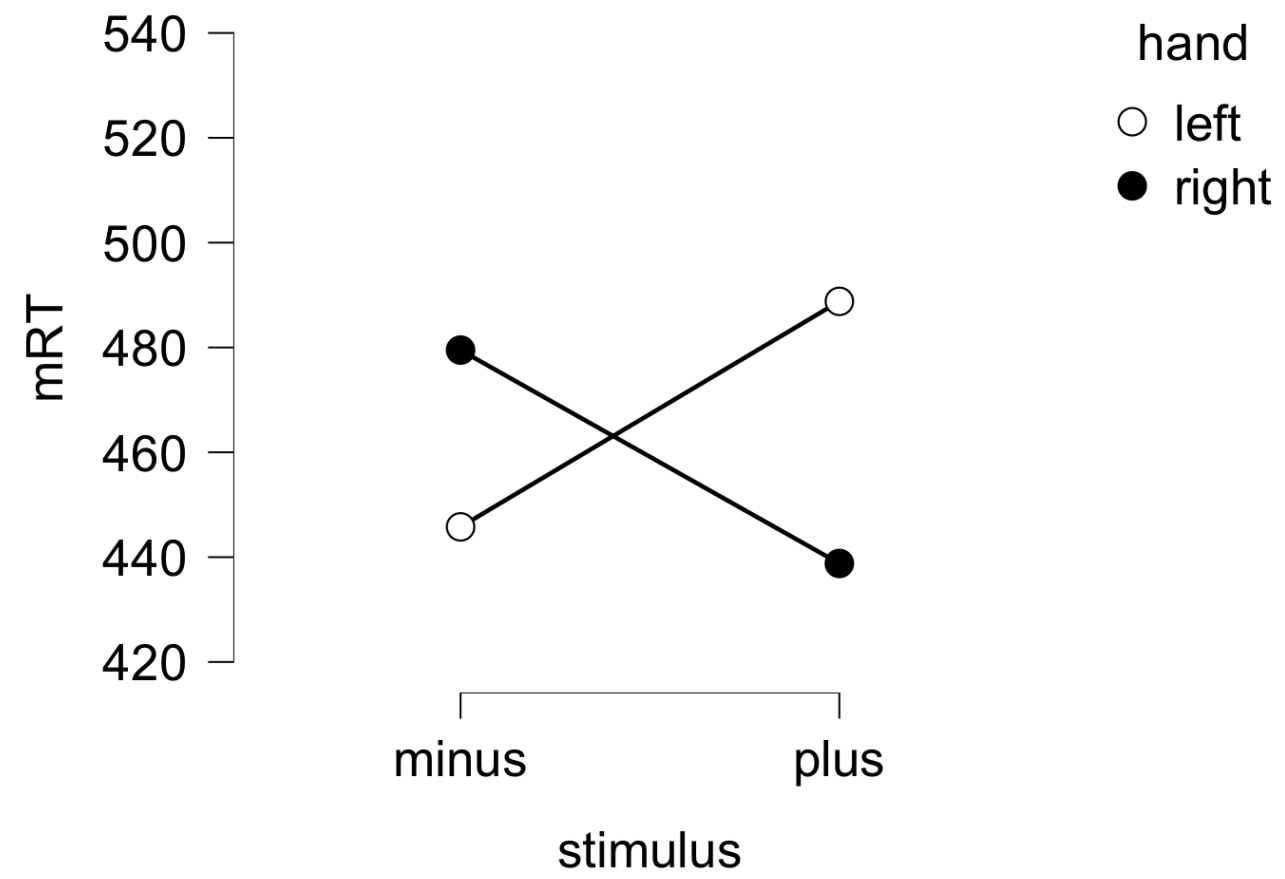

Figure 2. Mean response times (RTs) on the operation sign classification task, presented as a function of stimulus (plus sign or minus sign) and response hand (left, right).

\section{Bayesian t-test}

As a first step for the Bayesian $t$-test, we computed a difference score for each participant, defined by subtracting the mean response time for congruent trials from the mean response time for incongruent trials. We then performed a Bayesian $t$-test on these difference scores, giving a Bayes factor of $\mathrm{BF}_{+0}=10.28$ (see Figure 3). This Bayes factor indicates that the observed data are 10.28 times more likely under $\mathcal{H}_{+}$than under $\mathcal{H}_{0}$. Assuming 1-1 prior odds for $\mathcal{H}_{0}$ and $\mathcal{H}_{+}$, the observed data have increased the odds for $\mathcal{H}_{+}$ 
by a factor of 10.28 , giving a posterior probability of $\mathcal{H}_{+}$equal to $p\left(\mathcal{H}_{+} \mid\right.$data $)=0.91$. This is strong evidence for a positive OSSA effect.

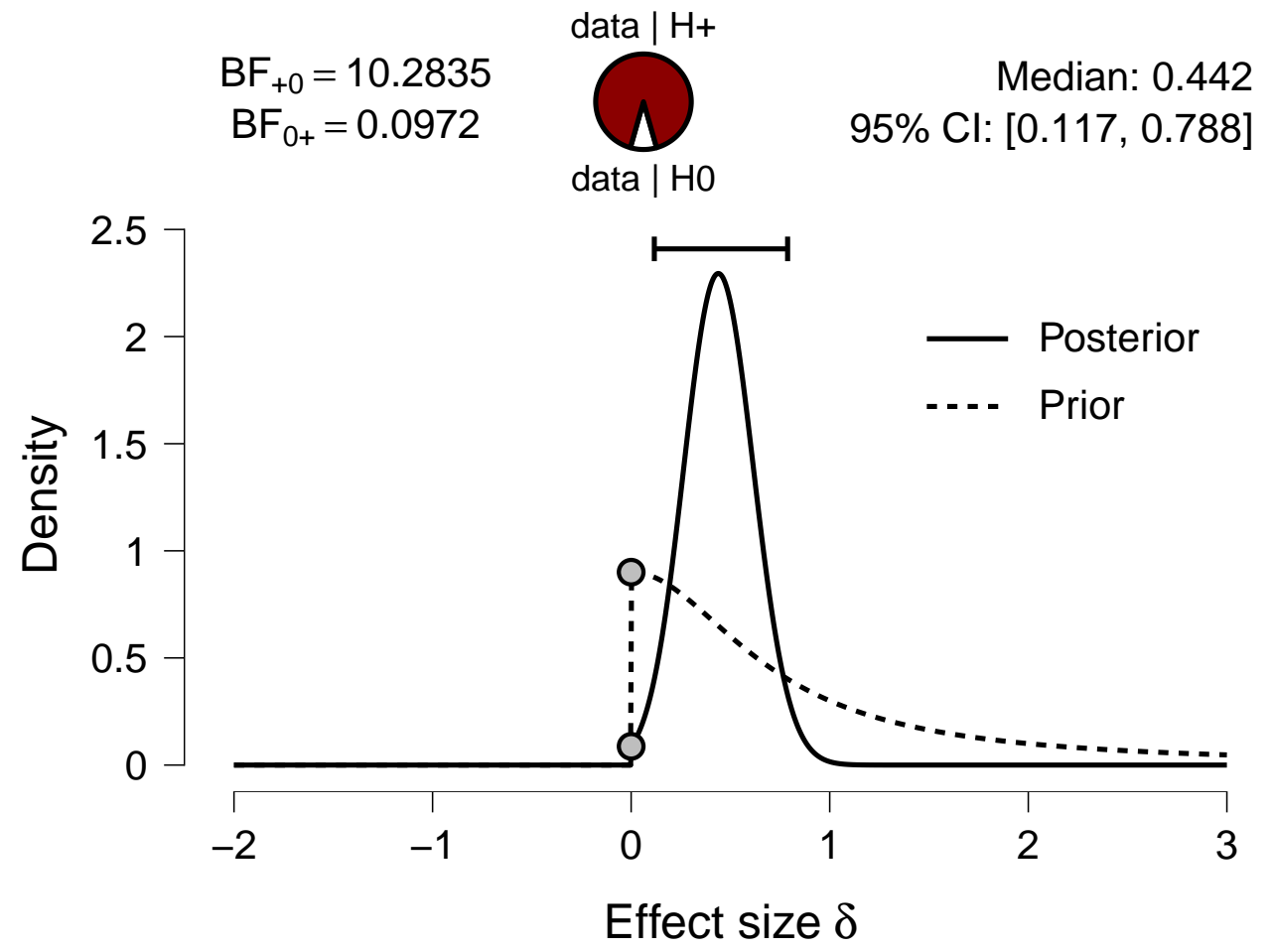

Figure 3. JASP output displaying prior and posterior distributions for the effect size $\delta$ under the hypothesis $\mathcal{H}_{+}: \delta>0$.

Now that we have evidence that the effect is positive (i.e., $\delta>0$ ), we may estimate the value of $\delta$, which gives us with a population-level estimate of the OSSA effect. Figure 3 shows how the observed data updated our prior belief about the effect size. The dashed line (the Cauchy prior) represents the probable values of effect size that we expected before observing data, and the solid line (the posterior) represents the distribution of probable values of updated after we observed the data. The figure shows that our belief that $\delta=0$ decreases after observing the data - note that the factor by which this decrease occurred is exactly $\mathrm{BF}_{+0}=10.28$. The median of the posterior distribution for $\delta$ was 0.442 , and our uncertainty about the size of the effect has shifted rightward so that $95 \%$ of the posterior distribution is between 0.117 and 0.788 . As $\delta$ represents a population-level version of Cohen's $d$, we can say with $95 \%$ probability that the OSSA effect is a medium-to-large effect.

\section{Sensitivity check}

The next step in our Bayesian analysis is to check the robustness of our claims under different prior settings. As explained above, the resulting Bayes factor, posterior probability for $\mathcal{H}_{+}$, and posterior distribution for $\delta$ depended on a specific choice of scale for the half-Cauchy prior on $\delta$. In Figure 4, we display a plot showing the values of $\mathrm{BF}_{+0}$ under a range of reasonable values of a priori scale for $\delta$. We can see that as the scale of the Cauchy prior increases, the values of $\mathrm{BF}_{+0}$ slightly decrease. The reason for this can be explained 
by again considering Figure 3. With a larger scale for the Cauchy prior (the dashed line), there will be slightly more mass on larger effects (i.e., the distribution will be more "spread out" and less peaked at 0 ). Thus, the factor by which the prior mass at $\delta=0$ decreases to the posterior mass at $\delta=0$ is smaller than before. As a result, the Bayes factor for $\mathcal{H}_{+}$is smaller. Specifically, we see that for a scale value of $r=1$ (a "wide" prior), we have a Bayes factor of $\mathrm{BF}_{+0}=8.53$, which converts to a posterior probability of $p\left(\mathcal{H}_{+} \mid\right.$data $)=0.895$. For a scale value of $r=\sqrt{2}$ (an "ultrawide" prior), we have a Bayes factor of $\mathrm{BF}_{+0}=6.65$, which converts to a posterior probability of $p\left(\mathcal{H}_{+} \mid\right.$data $)=0.87$. In both of these specific cases, we see that our observed data have given us good evidence for $\mathrm{H}+$ (i.e., a positive OSSA effect). Thus, our conclusions do not depend on the specific choice of prior scale for under $\mathcal{H}_{+}$.

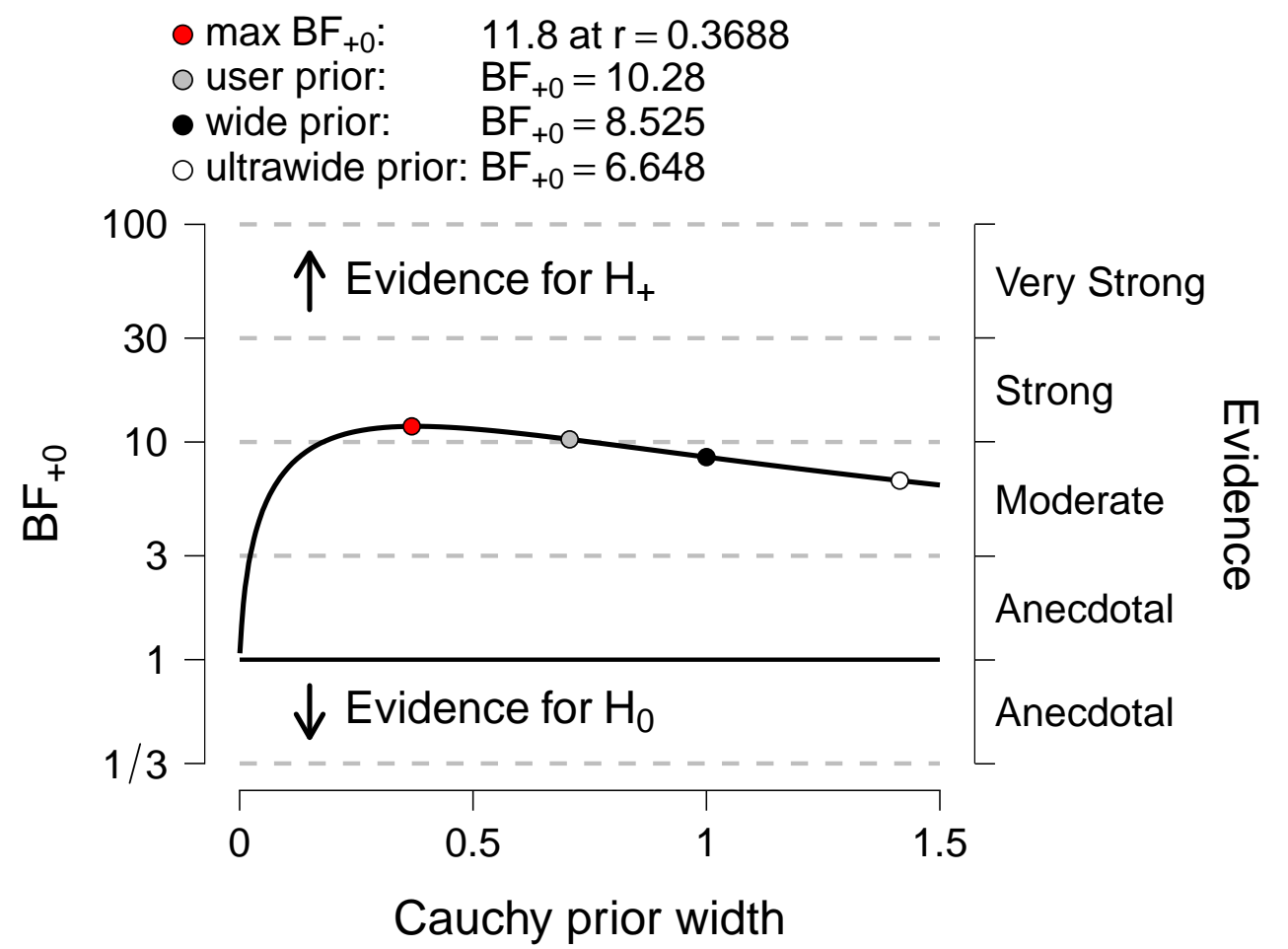

Figure 4. JASP output displaying a Bayes factors sensitivity check, where values of $\mathrm{BF}_{+0}$ are plotted against a range of different values of width (or scale) of the Cauchy prior for $\delta$.

\section{Sequential analysis}

Bayesian analysis also allows us to monitor the evidence as the incoming data is accumulated. In general, such sequential analysis is a strong advantage of Bayesian analyses over traditional approaches, as stopping data collection early once a certain threshold of evidence is achieved (i.e., "optional stopping") does not affect the interpretation of the resulting Bayes factor (Rouder, 2014). We have displayed this flow of evidence for our data in Figure 5. The general pattern shows increasing evidence for $\mathcal{H}_{+}$against $\mathcal{H}_{0}$ as the number of data points increases. For the first 10 or so data points, the data actually gives slight preference to $\mathcal{H}_{0}$. This simply reflects the principle of parsimony - when information is 
minimal, the Bayes factor will give preference to the simplest model $\left(\mathcal{H}_{0}\right)$. After 25 data points, the Bayes factor for $\mathcal{H}_{+}$increases above 3 , which provides a posterior probability for $\mathcal{H}_{+}$greater than 0.75. As data accumulates beyond $N=25$, the level of evidence for $\mathcal{H}_{+}$ continues to increase accordingly. As we can see, there would be little benefit to collecting a larger sample - with $N=35$, we have 10 -to- 1 posterior odds for $\mathcal{H}_{+}$. We think this is sufficient to provide strong support for a positive OSSA effect.

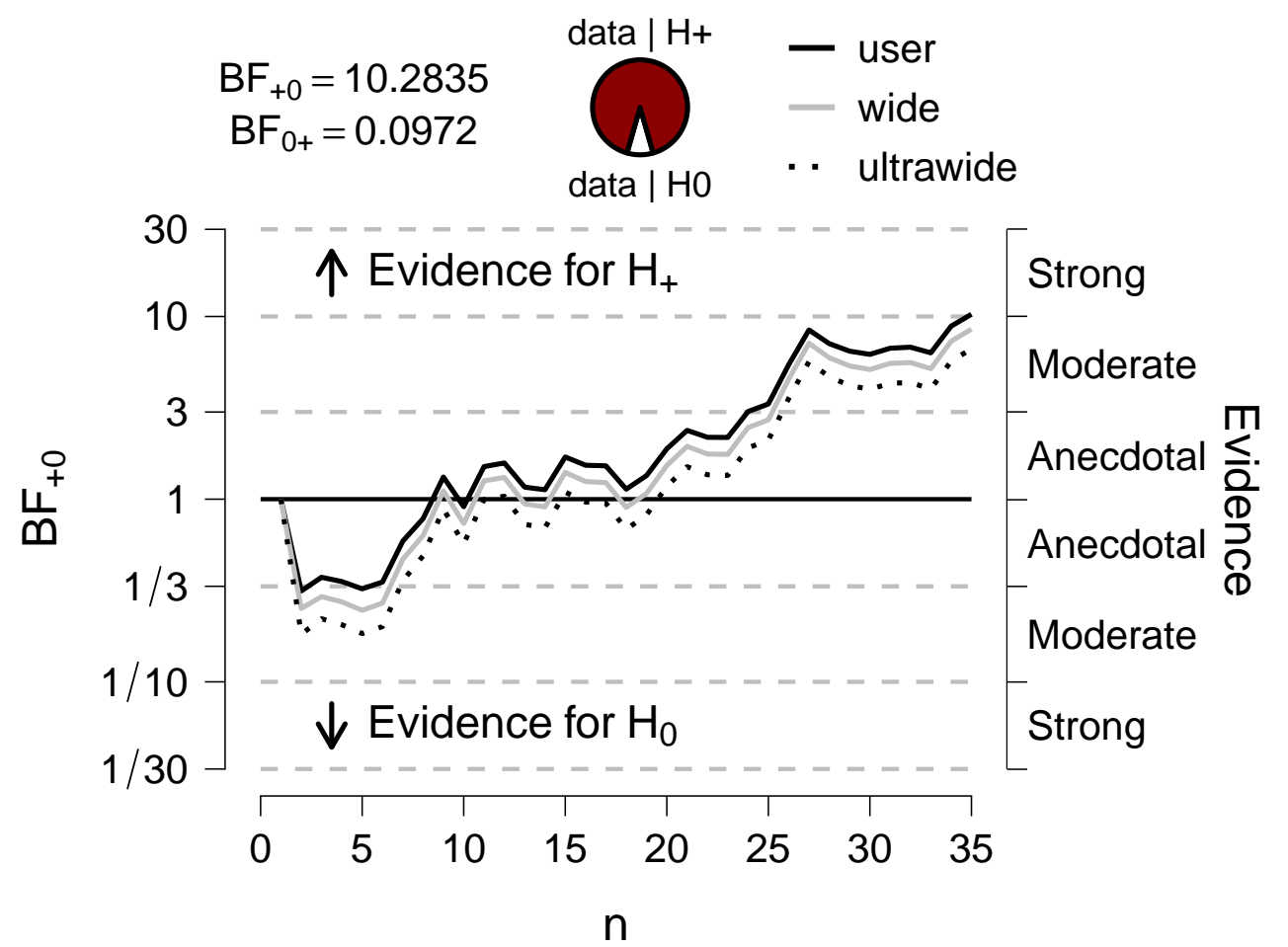

Figure 5. JASP output displaying a sequential analysis, showing the evidential flow of $\mathcal{H}_{+}: \delta>0$ versus $\mathcal{H}_{0}: \delta=0$ as data accumulates.

\section{Testing with an informed prior}

As a final analysis, we considered a Bayesian $t$-test with non-default prior specification. Though default priors are mathematically shown to perform well in an objective Bayesian sense (Rouder et al., 2009), one can argue that our a priori expectation about the population level effect size $\delta$ is not captured by the positive half-Cauchy distribution. As can be seen in Figure 1, the positive half-Cauchy has its greatest mass at $\delta=0$, which would indicate that our a priori belief that $\delta=0$ is greater than any other positive value of $\delta$. This "skeptics view" about the effect may be undesirable for some. What would happen if we were to choose a prior for that placed its greatest mass over a positive effect rather than on $\delta=0$ ?

To answer this question, we performed an informed Bayesian t-test (Gronau, Ly, \& Wagenmakers, 2019). Instead of using the positive half-Cauchy as a prior distribution for $\delta$, the informed $t$-test uses a prior distribution that matches the shape and scale of the collection of expected effect sizes elicited from expert consultation. One such informed prior that works well for a variety of situations in psychology is the Oosterwijk prior (Gronau et 
al., 2019), which has a median of 0.35 and 33rd and 66th percentile values of 0.25 and 0.45 , respectively. This prior is mathematically specified as a scaled noncentral $t$-distribution with location 0.35, scale 0.102, and $\mathrm{df}=3$ (see the right panel of Figure 1). Implementing the Oosterwijk prior in JASP gives a Bayes factor of $\mathrm{BF}_{+0}=25.40$ (see Figure 6). This equates to a posterior probability of $p\left(\mathcal{H}_{+} \mid\right.$data $)=0.96$. As we can see in Figure 6 , the informed prior places less mass on extreme effects than the default Cauchy, resulting in less variability in our estimate of $\delta$, as reflected by a $95 \%$ credible interval of $[0.20,0.61]$. Again, the take home message is that we have substantial evidence for a positive OSSA effect.

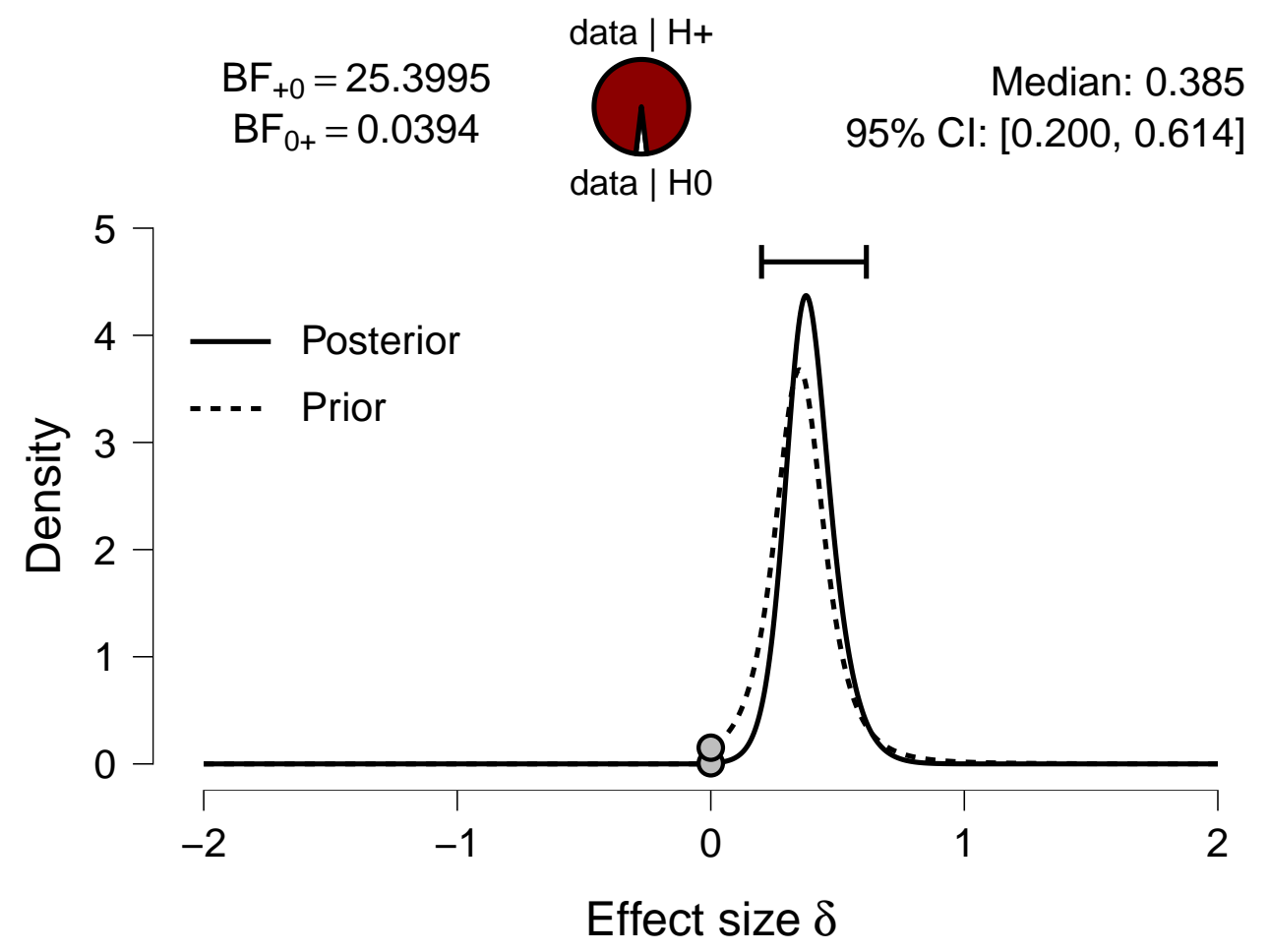

Figure 6. JASP output displaying prior and posterior distributions for the effect size under the hypothesis $\mathcal{H}_{+}: \delta>0$. This analysis uses the informed Oosterwijk prior, a scaled noncentral $t$-distribution with location 0.35 , scale 0.102 , and $d f=3$.

\section{Discussion}

The purpose of this study was to replicate and confirm the operation sign spatial association (i.e., the OSSA effect) originally reported by (Pinhas et al., 2014). In our study, participants were asked to make a speeded classification of the arithmetic operation (addition or subtraction) that was represented by two mathematical operation signs ( + or -$)$. When responses were spatially congruent (i.e., addition and subtraction mapped to the right hand and left hand, respectively), participants performed this classification more quickly than on spatially incongruent trials (i.e., addition and subtraction mapped to the left hand and right hand, respectively). These results match those originally presented by (Pinhas et al., 2014) and indicate that people may form an association between arithmetic signs and spatial location; specifically, the minus sign may evoke a leftward association, whereas the plus sign 
may evoke a rightward association.

Additionally, we demonstrated how to perform a complete confirmatory Bayesian analysis of the observed data in our experiment. Using the free software package JASP (Faulkenberry et al., 2020; JASP Team, 2020), we showed how to perform the key steps in a confirmatory Bayesian analysis. First, we constructed two competing hypotheses about the population-level standardized effect $\delta$ : a null hypothesis $\mathcal{H}_{0}$ and a positive-effect alternative hypothesis $\mathcal{H}_{+}$. We then performed a Bayesian $t$-test (Rouder et al., 2009) on participants' mean differences in RTs between incongruent and congruent trials. The $t$-test produced a Bayes factor of 10.3 , indicating that our observed data were approximately 10 times more likely under $\mathcal{H}_{+}$than under $\mathcal{H}_{0}$. These 10 -to- 1 posterior odds are equivalent to a posterior model probability of 0.91 for $\mathcal{H}_{+}$. Further, the $t$-test allowed us to estimate with $95 \%$ probability the value of $\delta$, which we saw was between 0.12 and 0.79 . In all, we have strong evidence for a medium-to-large OSSA effect, indicating that people indeed form associations between operation signs and spatial location.

The key mechanism in our model comparison was the Bayes factor (Faulkenberry, 2022; Kass \& Raftery, 1995), the computation of which requires the analyst to specify a prior for $\delta$ under $\mathcal{H}_{+}$. That is, before observing data, one must first specify a probability distribution that mathematically encodes the variability of effect sizes one expects to obtain in the experiment. We initially did this using default specifications (i.e., an objective Bayesian approach, Kass \& Wasserman, 1996) and employed the positive half Cauchy distribution with scale $1 / \sqrt{2}$. To test the robustness of our results to this prior specification, we performed two additional sensitivity checks. First, we recomputed Bayes factors obtained from different values of scale for the Cauchy distribution. In general, Bayes factors decreased slightly as scale increased, though all remained sufficiently evidential for a positive OSSA effect. Second, we performed an informed Bayesian $t$-test using the Oosterwijk prior (Gronau et al., 2019), a scaled noncentral $t$-distribution specifically chosen to reflect an a priori belief that if $\mathcal{H}_{+}$is the chosen model, then the effect size $\delta$ should be small to medium in size, centered at 0.35 , with $33 \mathrm{rd}$ and 66 th percentiles equal to 0.25 and 0.45 , respectively. This informed prior produced a Bayes factor for $\mathcal{H}_{+}$over 20 (and consequently, a posterior probability for $\mathcal{H}_{+}$over 0.96$)$. As a result, we are quite confident in the evidential value of our data and feel strongly that the OSSA effect is a real cognitive phenomenon worth further study.

To this end, we now speculate on some possible reasons why people should exhibit an association between operation signs and spatial location. One possibility is that operation signs evoke dynamic mental representations that are similar to moving one's attention on a mental number line (i.e., rightward for addition and leftward for subtraction). This view has gained traction in the literature (Marghetis \& Núñez, 2013; Marghetis et al., 2014) and reflects a core assumption of some recent theories about the nature of mental arithmetic (Fayol \& Thevenot, 2012; Mathieu, Gourjon, Couderc, Thevenot, \& Prado, 2016; Uittenhove, Thevenot, \& Barrouillet, 2016). Alternatively, the association may simply stem from longterm memory processes. For example, it may be the case that perceiving a plus sign activates a long-term memory representation of "adding", which in general results in quantities of larger magnitude. Similarly, a minus sign may activate representations of smaller magnitude. This may then work in concert with memory representations of conventional number lines 
being ordered in increasing magnitude from left to right to produce the observed association. Pinhas et al. (2014) also speculated about these two competing accounts of the OSSA effect and pointed out that while OSSA correlated positively with another task designed to capture operational momentum, it did not correlate with the SNARC effect. Because SNARC is often thought to reflect a mental number line (i.e., a long-term memory representation abstracting the general experience of seeing small numbers with the left side of space and large numbers with the right side of space), the lack of correlation between SNARC and OSSA calls into question any account for OSSA that is based on similar long-term memory processes.

Additionally, Pinhas et al. (2014) pointed out that the congruity effect was larger for the plus sign than for the minus sign, ruling out a polarity correspondence account often used to explain SNARC (e.g., Proctor \& Cho, 2006). We observed a similar pattern in Figure @ref\{fig:descriptives\}, but we did not explicitly test this. Pinhas et al. (2014) speculated that this asymmetry stems from the fact that the negative sign has multiple mathematical contexts in which it is typically used, and thus, it is more difficult to assign the negative sign to a particular spatial context. This would, in effect, attenuate the magnitude of the leftward operational momentum of the negative sign compared to the rightward momentum of the addition sign. Future studies could indeed test this claim by introducing a neutral condition to separate the congruity effect into facilitation and inhibition components, as is typically done in experiments with the size-congruity effect (e.g., Henik \& Tzelgov, 1982).

In summary, we successfully replicated the original observation by Pinhas et al. (2014) of an association between arithmetic operation signs and spatial location. Further, we described how to perform a confirmatory Bayesian analysis of the observed data using the free software package JASP. We conclude that there is substantial evidence for a positive OSSA effect. We believe that this confirmatory work situates operational-spatial associations as a serious line of inquiry in numerical cognition that is worthy of further exploration. Further, we hope that researchers can use the methods described here as a way to conduct their own confirmatory work. 


\section{References}

Azhar, M., Chen, Y., \& Campbell, J. I. D. (2020). Reading direction and spatial effects in parity and arithmetic tasks. Psychological Research. https://doi.org/10 .1007/s00426-020-01397-y

Chen, Y., \& Campbell, J. I. D. (2017). "Compacted" procedures for adults' simple addition: A review and critique of the evidence. Psychonomic Bulletin \& Review, 25(2), 739-753. https://doi.org/10.3758/s13423-017-1328-2

Cumming, G., \& Calin-Jageman, R. (2016). Introduction to the new statistics. Routledge. https://doi.org/10.4324/9781315708607

Dehaene, S., Bossini, S., \& Giraux, P. (1993). The mental representation of parity and number magnitude. Journal of Experimental Psychology: General, 122(3), 371-396. https://doi.org/10.1037/0096-3445.122.3.371

Dehaene, S., Dupoux, E., \& Mehler, J. (1990). Is numerical comparison digital? Analogical and symbolic effects in two-digit number comparison. Journal of Experimental Psychology: Human Perception and Performance, 16(3), 626-641. https://doi.org/10.1037/0096-1523.16.3.626

Faulkenberry, T. J. (2016). Testing a direct mapping versus competition account of response dynamics in number comparison. Journal of Cognitive Psychology, 28(7), 825-842. https://doi.org/10.1080/20445911.2016.1191504

Faulkenberry, T. J. (2019a). A tutorial on generalizing the default Bayesian $t$-test via posterior sampling and encompassing priors. Communications for Statistical Applications and Methods, 26(2), 217-238. https://doi.org/10.29220/csam.2019.2 6.2 .217

Faulkenberry, T. J. (2019b). Estimating evidential value from analysis of variance summaries: A comment on Ly et al. (2018). Advances in Methods and Practices in Psychological Science, 2(4), 406-409. https://doi.org/10.1177/2515245919872960

Faulkenberry, T. J. (2022). Psychological statistics: The basics. Routledge. https: //doi.org/10.4324/9781003181828

Faulkenberry, T. J., Ly, A., \& Wagenmakers, E.-J. (2020). Bayesian inference in numerical cognition: A tutorial using JASP. Journal of Numerical Cognition, 6(2), 231-259. https://doi.org/10.5964/jnc.v6i2.288

Fayol, M., \& Thevenot, C. (2012). The use of procedural knowledge in simple addition and subtraction problems. Cognition, 123(3), 392-403. https://doi.org/ 10.1016/j.cognition.2012.02.008

Fischer, M. H., \& Shaki, S. (2014). Spatial biases in mental arithmetic. Quarterly Journal of Experimental Psychology, 67(8), 1457-1460. https://doi.org/10.1080/ 17470218.2014.927516

Gronau, Q. F., Ly, A., \& Wagenmakers, E.-J. (2019). Informed Bayesian $t$-tests. The American Statistician, 74(2), 137-143. https://doi.org/10.1080/00031305.2 018.1562983

Henik, A., \& Tzelgov, J. (1982). Is three greater than five: The relation between physical and semantic size in comparison tasks. Memory \&6 Cognition, 10(4), 389-395. https://doi.org/10.3758/bf03202431

JASP Team. (2020). JASP (Version 0.14.1)[Computer software]. Retrieved from https://jasp-stats.org/ 
Kass, R. E., \& Raftery, A. E. (1995). Bayes factors. Journal of the American Statistical Association, 90(430), 773-795. https://doi.org/10.1080/01621459.199 5.10476572

Kass, R. E., \& Wasserman, L. (1996). The selection of prior distributions by formal rules. Journal of the American Statistical Association, 91 (435), 1343-1370. https://doi.org/10.1080/01621459.1996.10477003

Marghetis, T., \& Núñez, R. (2013). The motion behind the symbols: A vital role for dynamism in the conceptualization of limits and continuity in expert mathematics. Topics in Cognitive Science, 5(2), 299-316. https://doi.org/10.1111/tops.12013

Marghetis, T., Núñez, R., \& Bergen, B. K. (2014). Doing arithmetic by hand: Hand movements during exact arithmetic reveal systematic, dynamic spatial processing. Quarterly Journal of Experimental Psychology, 67(8), 1579-1596. https://doi.org/10.1080/17470218.2014.897359

Masson, M. E. J. (2011). A tutorial on a practical Bayesian alternative to nullhypothesis significance testing. Behavior Research Methods, 43(3), 679-690. https://doi.org/10.3758/s13428-010-0049-5

Mathieu, R., Gourjon, A., Couderc, A., Thevenot, C., \& Prado, J. (2016). Running the number line: Rapid shifts of attention in single-digit arithmetic. Cognition, 146, 229-239. https://doi.org/10.1016/j.cognition.2015.10.002

McCrink, K., Dehaene, S., \& Dehaene-Lambertz, G. (2007). Moving along the number line: Operational momentum in nonsymbolic arithmetic. Perception $\mathcal{G}_{3}$ Psychophysics, 69(8), 1324-1333. https://doi.org/10.3758/bf03192949

Pinhas, M., Shaki, S., \& Fischer, M. H. (2014). Heed the signs: Operation signs have spatial associations. Quarterly Journal of Experimental Psychology, 67(8), 1527-1540. https://doi.org/10.1080/17470218.2014.892516

Proctor, R. W., \& Cho, Y. S. (2006). Polarity correspondence: A general principle for performance of speeded binary classification tasks. Psychological Bulletin, 132(3), 416-442. https://doi.org/10.1037/0033-2909.132.3.416

Rouder, J. N. (2014). Optional stopping: No problem for Bayesians. Psychonomic Bulletin \& Review, 21 (2), 301-308. https://doi.org/10.3758/s13423-014-0595-4

Rouder, J. N., Speckman, P. L., Sun, D., Morey, R. D., \& Iverson, G. (2009). Bayesian $t$ tests for accepting and rejecting the null hypothesis. Psychonomic Bulletin \& Review, 16(2), 225-237. https://doi.org/10.3758/pbr.16.2.225

Thevenot, C., \& Barrouillet, P. (2020). Are small additions solved by direct retrieval from memory or automated counting procedures? A rejoinder to Chen and Campbell (2018). Psychonomic Bulletin 89 Review, 27(6), 1416-1418. https: //doi.org/10.3758/s13423-020-01818-4

Uittenhove, K., Thevenot, C., \& Barrouillet, P. (2016). Fast automated counting procedures in addition problem solving: When are they used and why are they mistaken for retrieval? Cognition, 146, 289-303. https://doi.org/10.1016/j.cognit ion.2015.10.008

Wagenmakers, E.-J. (2007). A practical solution to the pervasive problems of $p$ values. Psychonomic Bulletin \& Review, 14(5), 779-804. https://doi.org/10.375 8/bf03194105 